\title{
Measurement of linear velocity of laminar gas flow at different static pressure
} A. P. Tyukin, Candidate of Technical Sciences, Head of the Project Initiation Department of the Engineering Center ${ }^{1}$,
e-mail: TukinAP@yandex.ru

${ }^{1}$ Kola Mining and Metallurgical Company, Monchegorsk, Russia.

\begin{abstract}
Today, the issue of erecting building units on the surface of the Moon is gaining significant importance. The main problem is the cost of delivery of structural materials. The solution may be to obtain a structural material from regolith. The regolith from the Mare Crisium contains up to $20 \%$ of the amount of iron and titanium and is a polymetallic ore. Its concentration is possible by gas- dynamic separation using a gas in a light insulated chamber under low absolute pressure $(10-30 \mathrm{kPa})$. The processes of gas-dynamic separation with the use of rarefied gases are studied at the Department of Minerals Concentration of NUST MISIS, Moscow, Russian Federation.

The paper describes a barostatic research installation of gas-dynamic separation and the process of determining the true gas velocity at various values of its static pressure. The authors have calculated the air velocity according to hydrodynamic formulas, and the true air velocity in the acceleration channel of the separator was established as well with the use of special equipment. A comparison of calculated and experimental data was carried out and a high convergence was ascertained: the difference was no more than $20 \%$ rel. Resulting from the calculations and experiments, the principles on the basis of which the gas velocity setting and control should be carried out during studies on gas-dynamic separation are formulated.
\end{abstract}

Key words: the Moon, regolith, concentration, gas-dynamic, separation, velocity, measurement.

DOI: $10.17580 / \mathrm{nfm} .2021 .02 .02$

\section{Introduction}

S tates and leading space agencies are developing the Moon exploration programs [1-2]. In this regard, the issue of constructing building units on the lunar surface may acquire significant importance in the near future. At the same time, the main problem is the high cost of delivering structural materials from the Earth. This problem may be solved by obtaining a structural material from regolith.

In particular, the regolith from the Mare Crisium contains up to $20 \%$ of the amount of iron and titanium and so it is a polymetallic ore. Regolith concentration is possible by gas-dynamic separation using a gas located in a light insulated chamber under low absolute pressure $(10-30 \mathrm{kPa})$.

The processes of gas-dynamic separation with the use of rarefied gases are studied at the Department of Minerals Concentration of NUST MISIS, Moscow, Russian Federation. The paper describes a barostatic research installation for gas-dynamic separation of granular materials, as well as the process of determining the true gas velocity using the example of air at different values of its static pressure and at various compressor drive power.

The authors have measured the pressure differential in the supercharger receiver and the main volume of a vacuum compression chamber; the air velocity according to the equations of hydrodynamics was calculated. With the use of special equipment, a high-speed shooting (960 frames/s) of tracer particles of light material fed into the separator and acquiring a speed close to own air velocity was performed. Based on the results of the analysis of video sequences, the actual air velocity is calculated for various operating parameters.

The calculated and experimental results were compared and their high convergence was established: the difference is not more than $20 \%$ rel. At the same time, predicted deviations were fixed at low pressures (at the level of $20 \mathrm{kPa}$ ) and at high values of the Reynolds number (more than 7000).

Based on the results of implemented calculations and experimental work, the main principles basing on which the gas velocity setting and subsequent control should be carried out when conducting studies on the gas-dynamic separation of granular materials are formulated.

\section{Setting up the real-world problem}

In the near future, the issue of erecting building structures on the Moon surface may become significant. Stations for people to work, wireless transmitters-repeaters, remotely controlled telescopes should be noted among the possible construction objects [3]. For example, a long-range telescope of any range built on the lunar surface would be much more efficient than its Ground-based counterpart and significantly cheaper than the orbital version.

The main problem on the way to progress in this direction will be the delivery of structural (building) materials from the Ground. Now, the cost of delivering $1 \mathrm{~kg}$ of cargo from the Earth to the Moon is so high that the construction of objects on the lunar surface does not generate real interest among either states or private companies. 
To solve this problem may obtaining the structural material from existing raw materials - regolith - by the methods available for autonomous implementation without the use of water, reagents, maintenance staff and applying practically unlimited solar radiation energy [4].

For example, the regolith delivered to the Earth in 1976 by Luna 24 automatic interplanetary station contains the components listed in Table 1 [5].

The maximum iron and titanium contents are 19.1 and $1.4 \%$, respectively. In fact, regolith is a polymetallic ore already prepared for concentration - broken and crushed. The task is reduced to the required size class separation by means of unmanned self-cleaning sieves.

In conditions of vacuum and surface temperature fluctuations, the amplitude of which reaches $300 \mathrm{~K}$, the use of classical beneficiation methods implemented in an aqueous medium (flotation, wet magnetic separation) is not possible. In this case, it is advisable to consider dry separation methods based on differences of mineral particles in density, size, shape, magnetic, electrical or other properties that become apparent without the use of water [6].

One of the possible ways to separate particles by density and shape (within the selected size class) is gas-dynamic separation in a laminar gas flow. The use of a slotted acceleration channel makes it possible to significantly reduce the hydraulic diameter of the acceleration channel and ensure a laminar gas flow regime, and its fixed limited length allows the gas flow dynamic pressure to have an effect upon the particles for a managed time and eventually give them different rates depending on their properties. Heavy particles, as well as the particles with a lower coefficient of aerodynamic resistance, will acquire a lower speed by the time they exit the acceleration channel and then, after a short movement along the ballistic trajectory, will be captured separately [7].

In practice, a gas-dynamic separator is a device that requires gas supply at a constant speed to operate. The compressor pumps the gas into the receiver, designed to compensate for pressure fluctuations, and then the gas flows through pipelines to the rear part of the main element of the separator - the acceleration channel. Feeding the separated mixture into the acceleration channel is carried out through a hole in the upper part of the channel using a feeder.

In order to avoid unwanted gas leakage through the feeder, the latter is enclosed in a sealed housing. Consequently, the gas-dynamic separation process is periodic, and it has to be stopped when the next volume of the separated mixture needs to be loaded into a sealed feeder.

Because the proposed method of regolith concentration involves the use of a gas as a working medium, the separator must be placed into a sealed shell to avoid gas leakage into outer space. The use of a gas-dynamic separator on the surface of the Moon with an external sealed gas filled shell is worthwhile only if such a shell can be made of light material delivered from the Earth. Ideally, it is made of soft elastic material with supporting elements.
Table 1

Partial composition of the regolith from the Mare Crisium

\begin{tabular}{|c|c|c|c|c|}
\multirow{2}{*}{ Component } & \multicolumn{4}{|c|}{ Phase } \\
\cline { 2 - 5 } & Gabbro & Basalt & Dolerite & Anorthosite \\
\hline $\mathrm{TiO}_{2}$ & up to 0.7 & $0.7-2.3$ & $1.0-1.2$ & - \\
\hline $\mathrm{Al}_{2} \mathrm{O}_{3}$ & up to 19.0 & $11.1-17.2$ & $12.4-13.9$ & - \\
\hline $\mathrm{FeO}$ & $14.1-18.5$ & $15.8-24.6$ & $18.4-24.6$ & up to 3.3 \\
\hline
\end{tabular}

With a static gas pressure equal to the pressure of the Earth's atmosphere (101325 Pa), such a task is not feasible in practice.

According to the author's experience, an industrial separator with a minimum volume operating on a carrier gas at a pressure $100 \mathrm{kPa}$ higher than the ambient pressure requires a pressure chamber made of a steel sheet with a thickness of at least $8 \mathrm{~mm}$. The construction of such a structure from the materials delivered from the Earth would reduce to zero the original problem put by.

At the same time, effective gas-dynamic separation is possible at an absolute static pressure of the working environment about $10-30 \mathrm{kPa}$, which already allows such a process to be realized under ambient vacuum without the use of a heavy pressure chamber.

The processes of gas-dynamic separation of mineral materials under conditions of low absolute static pressures of the working environment are studied at the Department of Concentration and Processing of Minerals and Technogenic Raw Materials of the National Research Technological University "MISIS" (Moscow, Russian Federation). For this purpose, a barostatic research facility with a vacuum-compression pressure chamber has been designed and built as the key element inside which a gasdynamic separator is placed.

\section{Description of the research plant}

General view of the research plant is shown in Fig. 1.

The schematic diagram of the research plant design is shown in Fig. 2.

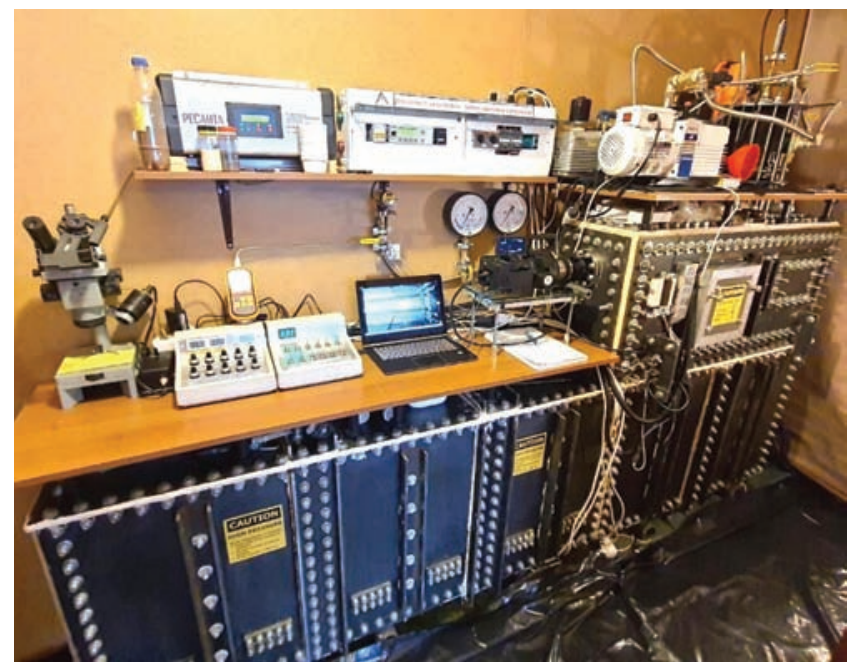

Fig. 1. General view of the barostatic research plant 


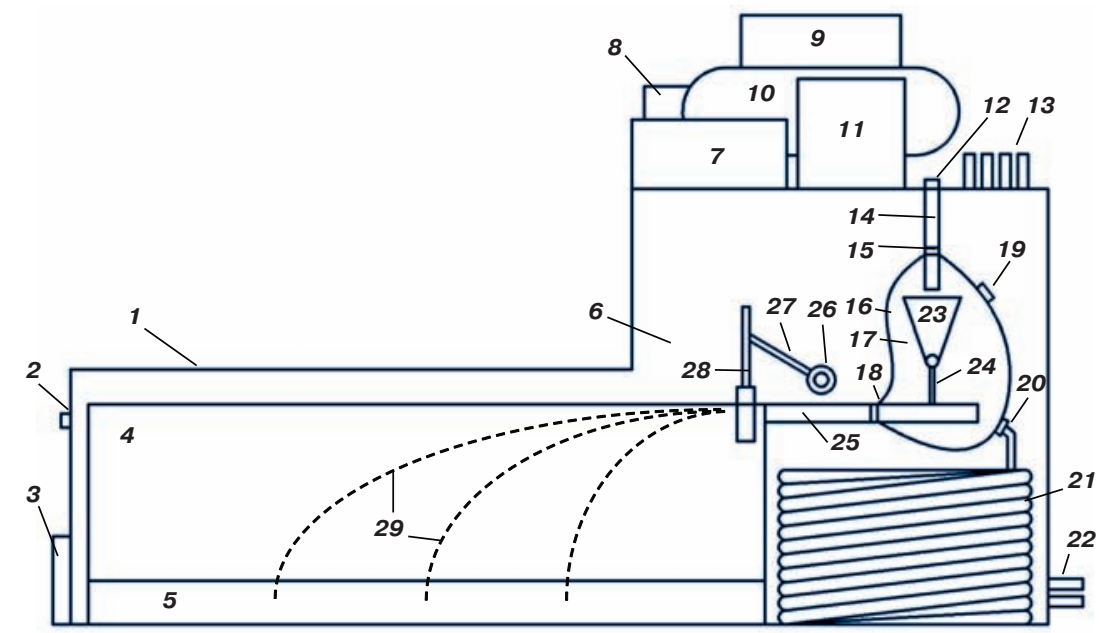

Fig. 2. The schematic diagram of the barostatic research plant for gas-dynamic separation: 1 - vacuum compression chamber; 2 - nipple cover for entering cleaning tools; 3 - process hatch for unloading separation products; 4 - bumper glasses limiting the dispersion of particles along the transverse axis; 5 - receiving containers for separation products; $6-$ the area under vacuum or high pressure during plant operation; $7-$ vacuum-pump No. 1;8 - vacuum-pump No. 2; 9-pressure boosting compressor; 10 - receiver of the pressure boosting compressor; 11 - inlet filter of vacuum-pumps; 12 - nipple cover for feed supply; 13 - inlets of pressure boosting compressor and vacuum-pumps; 14 - feeding chute; 15 - fixing point of the elastic receiver shell to the power supply loading heat; 16 - elastic receiver shell; $17-$ the area of increased pressure during separator operation; 18 - fixing point of the elastic receiver shell to the acceleration channel; 19 - cable entry node into the elastic receiver shell; $20-$ inlet from the separator compressor into the elastic receiver shell; 21 - working compressor for pumping the working medium into the acceleration channel and liquid heat exchanger for compressor cooling; 22 - input/output of the cooling liquid; 23 - service bunker and feeding worm; 24 - chute for feeding supply to the acceleration channel; 25 - acceleration channel; 26 - rotation axis of the anemometer holder; 27 - anemometer holder; 28 - anemometer; 29 - conventional ballistic trajectories of particles after exiting the acceleration channel

\section{Choosing a method for determining the gas velocity}

Under normal conditions, gas velocities are measured using anemometers. At the same time, the conditions under consideration differ in the following aspects:

- the height of the slotted acceleration channel is $1.6 \mathrm{~mm}$, which does not allow the use of anemometers with an impeller and ready-for-service devices with a heated string in the housing;

- the absolute static air pressure is in the range from 20 to $100 \mathrm{kPa}$, which would entail the need to calibrate the anemometer even if measuring air velocity with its help was possible;

- abrasive mineral particles move in the acceleration channel in the operating mode, which makes it impossible to install a stationary heated string inside the acceleration channel.

Thus, there are two possible ways to determine the gas velocity in the acceleration channel:

1. Installation of an anemometer of any design at a precisely fixed distance opposite the outlet slit of the acceleration channel, calibration of the device according to the true gas velocity at different compressor power and static gas pressure in the pressure chamber.

2. Determination of gas velocity by a pre-compiled matrix of velocity values at different compressor power and static gas pressure in the pressure chamber.

Both methods imply preliminary determination of the true gas velocity. It should be noted that a fast and accurate method for the gas velocity determining is necessary, among other things, for mathematical modeling of the gas-dynamic separation processes [8].

\section{Calculation of gas velocity by pressure difference}

Calculating the air velocity in the acceleration channel is a hydrodynamics problem about media outflow through a pipe from a container with the pressure $p_{0}$ into an area with pressure $p_{2}$. Schematically, this task is presented in Fig. 3.

Let us draw a 1-1 section at the beginning of the acceleration channel and a 2-2 section at the outlet. For these sections, the Bernoulli equation is as follows:

$$
\begin{aligned}
& \rho g z_{1}+p_{1}+\frac{a_{1} V_{1}^{2}}{2} \cdot \rho= \\
& =\rho g z_{2}+p_{1}+\frac{a_{2} V_{2}^{2}}{2} 2 \cdot \rho+\Delta p .
\end{aligned}
$$

We will draw the comparison plane along the acceleration channel axis, then $z_{1}=z_{2}=0$.

The pressure in the 1-1 section will be in the first approximation considered equal to the pressure in the receiver, measured by a vacuum gauge.

The mass air flow rate in the acceleration channel is determined by formula [9]:

$$
G=f \cdot \sqrt{\frac{\rho_{1} \cdot\left(p_{1}^{2}-p_{2}^{2}\right) \cdot d}{p_{1} \cdot \lambda \cdot L}},
$$

where $f$ is the cross-sectional area of the acceleration channel, $\mathrm{m}^{2} ; d$ is a hydraulic diameter of the acceleration channel, $m ; \lambda$ is the coefficient of hydraulic friction; $\rho_{1}$ is the air density in the 1-1 section.

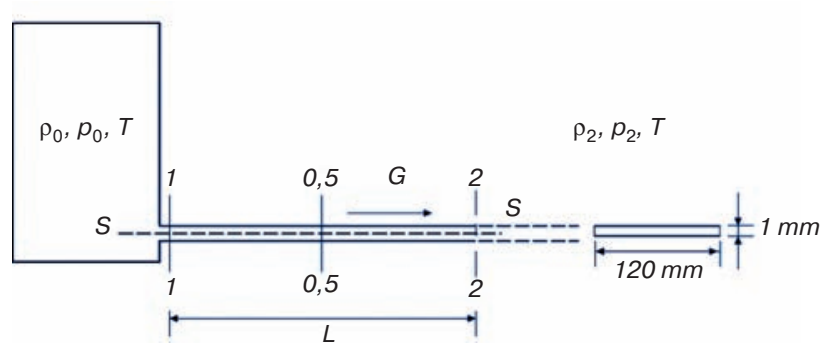

Fig. 3. Diagram of a typical problem about the flow of medium from a container through a pipe 
The coefficient of hydraulic friction is calculated according to the Weymouth formula [10]:

$$
\lambda=\frac{0.009407}{\sqrt[3]{d}} .
$$

Average air velocity at the beginning of the airway (from the continuity equation):

$$
V_{\mathrm{i}}=\frac{G}{\rho_{1} \cdot f} \text {. }
$$

According to the found airflow rate, the pressure loss at the inlet of the acceleration channel is calculated by the formula:

$$
\Delta p_{\text {in }}=\varsigma_{\text {in }} \cdot \frac{V_{1}^{2}}{2} \cdot \rho_{1},
$$

where $\varsigma_{\text {in }}=0.5$ is the inlet resistance coefficient [11-12].

Pressure in the 1-1 section in the second approximation:

$$
p_{1}^{(2)}=p_{0}-\Delta p \text {. }
$$

If the discrepancy between $p_{1}^{(1)}$ and $p_{1}^{(2)}$ does not exceed $1 \%$ rel., the accuracy of calculations can be considered acceptable. After that, the mass airflow rate is specified taking into account the correction for pressure loss.

The pressure in a $0.5-0.5$ section, which is the average in the acceleration channel, is calculated by the formula:

$$
p_{0.5}=\sqrt{p_{1}^{2}-\frac{G^{2}}{f^{2}} \cdot \frac{p_{1} \cdot \lambda \cdot 0.5 \cdot L}{\rho_{1} \cdot d}} .
$$

In turn, the air density is determined by the formula:

$$
\rho=\frac{p M}{R T},
$$

where $p$ is the air pressure, $\mathrm{Pa}, M$ is the air molar mass, $\mathrm{g} / \mathrm{mol}$; $R$ is the universal gas constant; $T$ is the air temperature, $\mathrm{K}$.

Based upon the improved values of the density and mass air consumption, the refined value of its density and, accordingly, linear velocity is calculated.

By the above procedure, the average linear air velocity in the acceleration channel is calculated at different values of static air pressure in the main volume. For this purpose, the static air pressure in the main volume of the pressure chamber and in the receiver was measured, as well as the air temperature at the outlet of the acceleration channel. According to these data and taking into account actual geometric parameters of the acceleration channel $(L=600 \mathrm{~mm}, B=$ $=120 \mathrm{~mm}, H=1.6 \mathrm{~mm}$ ), calculations of the air velocity values in the acceleration channel for each of eight combinations of static air pressure in the main volume of the pressure chamber and power consumption of the separator compressor drive values have been implemented. The calculation results are presented in Table 2 and Figs. 4, 5.

It can be seen that the calculated air velocity increases as the power consumed by the compressor increases, as well as with a decrease in static air pressure and, as a consequence, its density and viscosity.
Table 2

Calculated values of the average air velocity in the acceleration channel

\begin{tabular}{|c|c|c|}
$\begin{array}{c}\text { Absolute pressure } \\
\text { in the pressure chamber, } \mathrm{kPa}\end{array}$ & $\begin{array}{c}\text { Compressor } \\
\text { power, } \mathrm{kW}\end{array}$ & $\begin{array}{c}\text { Design } \\
\text { air velocity, m/s }\end{array}$ \\
\hline 20.0 & 0.2 & 21.0 \\
\hline 20.0 & 0.4 & 28.0 \\
\hline 60.0 & 0.3 & 13.3 \\
\hline 60.0 & 0.7 & 18.3 \\
\hline 60.0 & 1.1 & 21.5 \\
\hline 96.5 & 0.4 & 10.5 \\
\hline 96.5 & 0.9 & 15.2 \\
\hline 96.5 & 1.4 & 18.2 \\
\hline
\end{tabular}

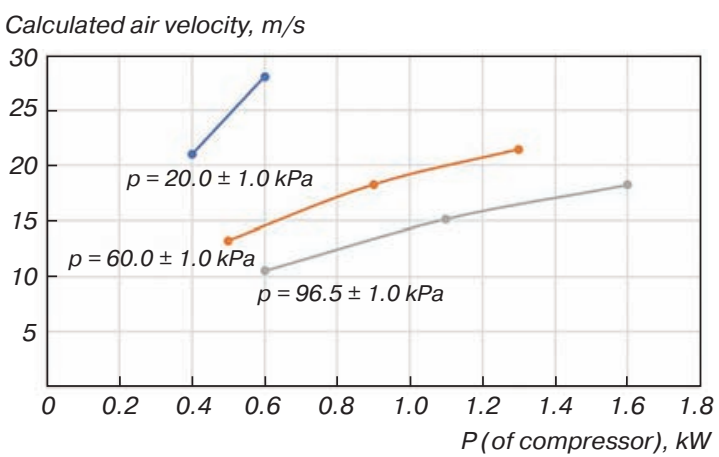

Fig. 4. Dependence of the calculated average air velocity in the acceleration channel $(\mathrm{m} / \mathrm{s})$ on power consumption of the separator compressor drive at different absolute values of static air pressure in the main volume of the pressure chamber
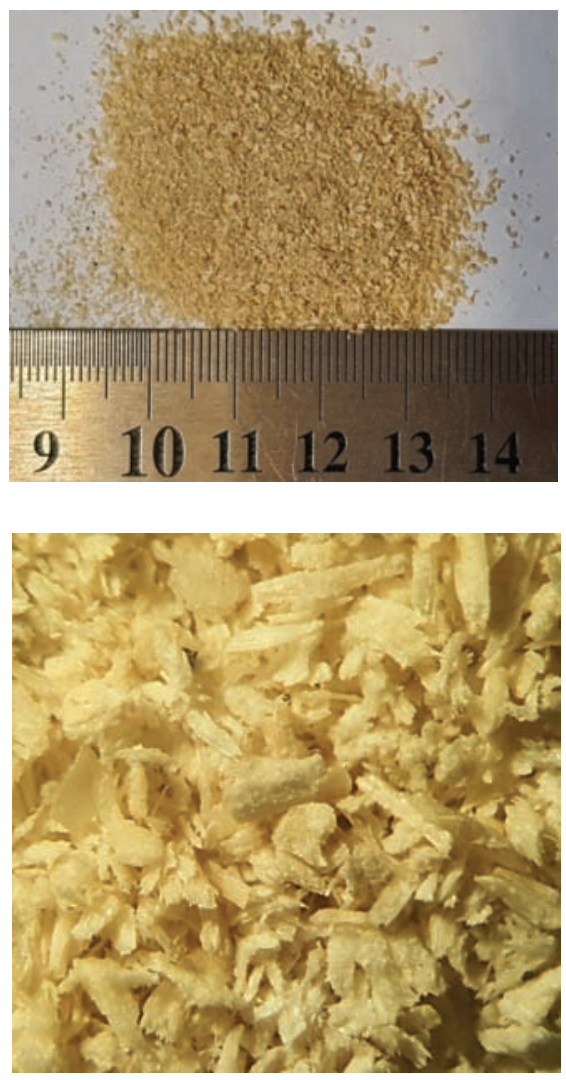

Fig. 5. Tracing material based on cellulose 


\section{Direct measurement of the true gas velocity}

In addition to the calculations, actual air velocity was directly measured at the same values of its static pressure in the main volume of the vacuum compression chamber and drive power of the separator compressor. The analysis of the high-speed video recording of the movement of light tracer particles in the acceleration channel was chosen as a measurement method.

For this purpose, a mixture of light particles, obviously acquiring linear velocity close to the own air velocity, has been fed in advance to the service bunker at a significant distance from the acceleration channel outlet. Such a material is cellulose of natural origin based on pine chips having an irregular shape, a sphericity coefficient less than 0.1 and, therefore, high windage, as well as a density of no more than $500 \mathrm{~kg} / \mathrm{m}^{3}$ (Fig. 5). A granulometric class of $-630+200 \mu \mathrm{m}$ was previously selected using metal sieves with a square cell.

The acceleration channel is transparent, since it is made of the 8 -mm-thick quartz glass. The velocity of tracer particles in the acceleration channel has been measured by high-speed shooting with a frame rate equal to 960 frames/s using a specialized Sony NEX-FS700K video camera. When shooting, a shutter speed of $1 / 1000 \mathrm{~s}$, an aperture number of $1 / 8$ and a photosensitivity of 1600 were set according to ISO 12232:2019 [13]. Especially for this series of experiments, a viewport was made on the front end of the vacuum compression chamber, and a mirror was installed inside, above the horizontally positioned acceleration channel. The equipment arrangement is shown in Fig. 6.

One of the critical aspects of highspeed shooting is lighting. First, the lighting should be powerful enough for shooting with a low shutter speed (in our case it is 960 frames per second with a shutter speed of $1 / 1000 \mathrm{~s}$ for each frame). Second, it is necessary to intensify the illumination of the studied objects and at the same time minimize the lightning of background objects to create the hardest image, because high-speed shooting is carried out at the maximum values of the photomatrix photosensitivity and the picture contrast affects the subsequent distinguishability of its details.

In the series of experiments under consideration, the author used two LED floodlights with a power of $40 \mathrm{~W}$ each, with a total power of $80 \mathrm{~W}$ (the equivalent power of an incandescent lamp is $600-800 \mathrm{~W}$ ), with a focused beam of neutral white light, as lighting devices. Black felt fabric has been used as a background when shooting to ensure maximum contrast and the floodlights has been located exactly opposite the outlet slit of the acceleration channel at a distance of $200 \mathrm{~mm}$ from it, so the focused beam of light is directed horizontally against the movement of particles (Fig. 7). At that, the angle between the light beam and plane of the acceleration channel is close to $0^{\circ}$, therefore, the light from the glass reflection coefficient is close to 1 and background lightning is practically not carried out.

The distance at which the floodlights are installed (200 $\mathrm{mm}$ ) is chosen because it is necessary, on the one hand, to place them as close as possible to provide intense lighting, and on the other hand, not to create obstacles to the air movement in the acceleration channel. The distance is chosen as the minimum at which there is no difference in the anemometer readings (see Fig. 7, in the center) in the presence or absence of installed floodlights.

Table 3 and Fig. 8 show the values of the pressure differential between the internal volume of the receiver and the main volume of the pressure chamber at different

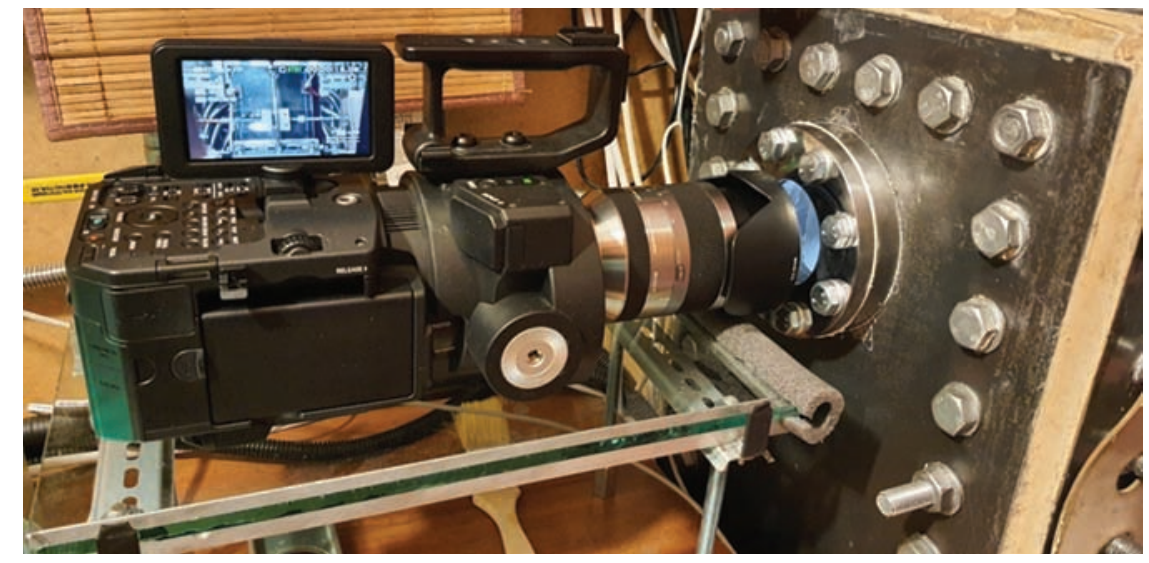

Fig. 6. A high-speed camera directed on the viewport of the third section of the vacuum compression chamber

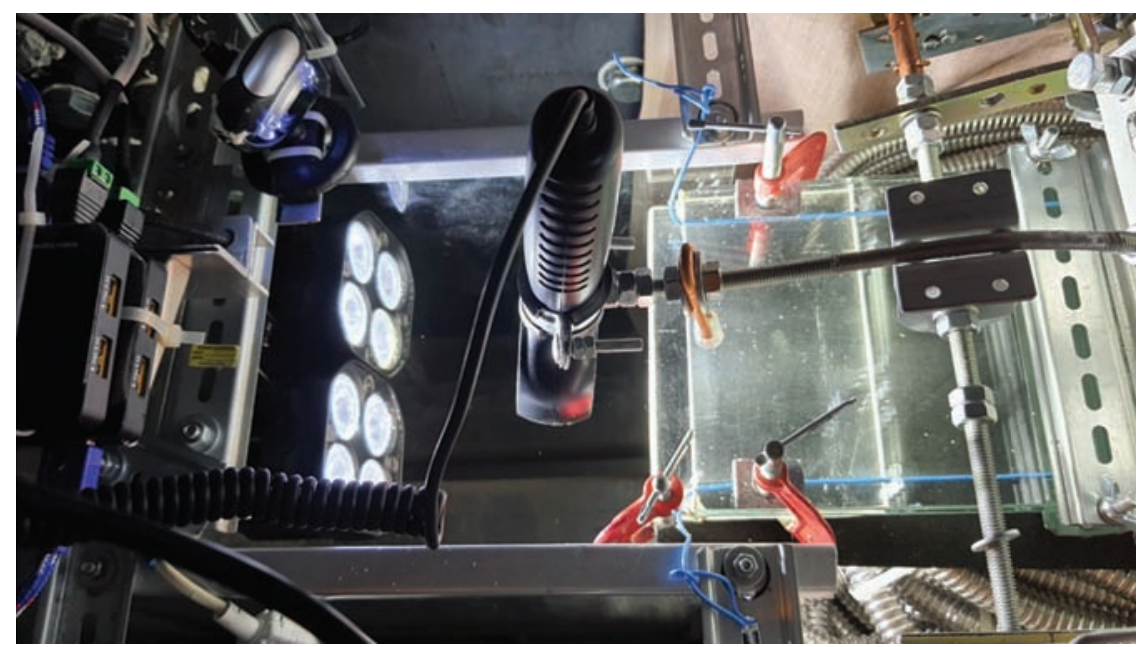

Fig. 7. LED floodlights installed opposite the acceleration channel outlet slit (in the center - the reference anemometer impeller) 
Table 3

Pressure differential between the internal volume of the receiver and the main volume of the pressure chamber

\begin{tabular}{|c|c|c|c|}
$\begin{array}{c}\text { Experiment } \\
\text { number }\end{array}$ & $\begin{array}{c}\text { Absolute pressure } \\
\text { in the pressure } \\
\text { chamber, kPa }\end{array}$ & $\begin{array}{c}\text { Compressor } \\
\text { power, kW }\end{array}$ & $\begin{array}{c}\text { Pressure } \\
\text { differential, Pa }\end{array}$ \\
\hline 1 & $20.0 \pm 1.0$ & 0.2 & 634.4 \\
\hline 2 & $20.0 \pm 1.0$ & 0.4 & 1155.0 \\
\hline 3 & $60.0 \pm 1.0$ & 0.3 & 765.6 \\
\hline 4 & $60.0 \pm 1.0$ & 0.7 & 1463.1 \\
\hline 5 & $60.0 \pm 1.0$ & 1.1 & 2039.4 \\
\hline 6 & $96.5 \pm 1.0$ & 0.4 & 776.9 \\
\hline 7 & $96.5 \pm 1.0$ & 0.9 & 1627.5 \\
\hline 8 & $96.5 \pm 1.0$ & 1.4 & 2363.1 \\
\hline
\end{tabular}

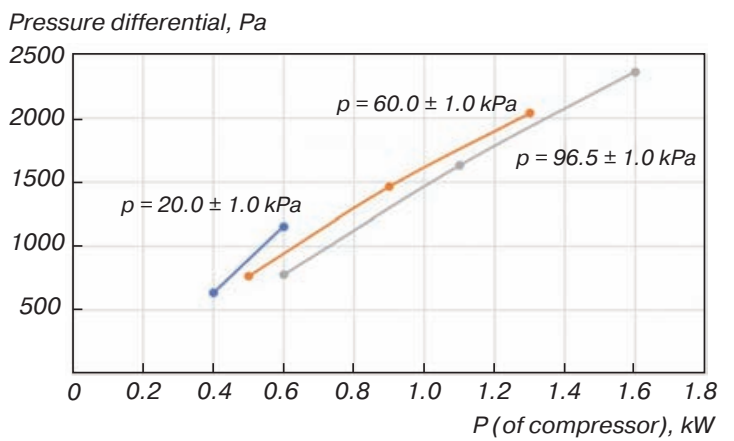

Fig. 8. The dependence of the pressure differential between the internal volume of the separator receiver and main volume of the pressure chamber $(\mathrm{Pa})$ on the drive power of the separator compressor $(\mathrm{kW})$ at different absolute values of static air pressure in the main volume of the pressure chamber

Table 4

Anemometer readings

\begin{tabular}{|c|c|c|c|}
$\begin{array}{c}\text { Experiment } \\
\text { number }\end{array}$ & $\begin{array}{c}\text { Absolute pressure } \\
\text { in the pressure } \\
\text { chamber, kPa }\end{array}$ & $\begin{array}{c}\text { Compressor } \\
\text { power, kW }\end{array}$ & $\begin{array}{c}\text { Anemometer } \\
\text { readings, m/s }\end{array}$ \\
\hline 1 & $20.0 \pm 1.0$ & 0.2 & 0.00 \\
\hline 2 & $20.0 \pm 1.0$ & 0.4 & 2.30 \\
\hline 3 & $60.0 \pm 1.0$ & 0.3 & 1.46 \\
\hline 4 & $60.0 \pm 1.0$ & 0.7 & 2.28 \\
\hline 5 & $60.0 \pm 1.0$ & 1.1 & 2.86 \\
\hline 7 & $96.5 \pm 1.0$ & 0.4 & 1.48 \\
\hline 8 & $96.5 \pm 1.0$ & 0.9 & 2.72 \\
\hline
\end{tabular}

Anemometer readings, $\mathrm{m} / \mathrm{s}$

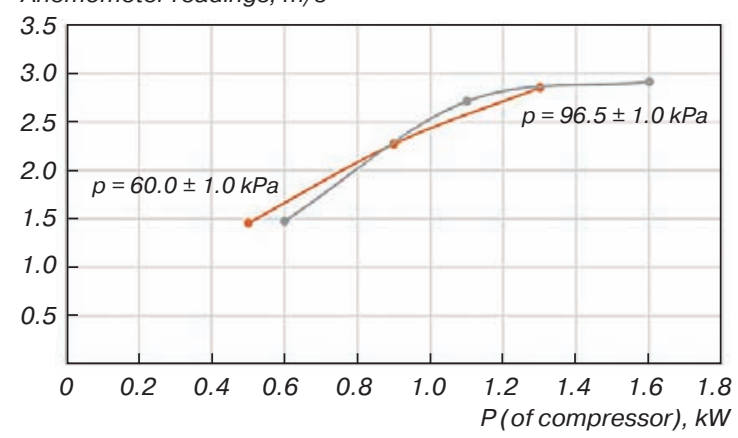

Fig. 9. Dependence of anemometer readings $(\mathrm{m} / \mathrm{s})$ on the drive power of the separator compressor $(\mathrm{kW})$ at different absolute values of static air pressure in the main volume of the pressure chamber values of the drive power of the separator compressor and static air pressure inside the pressure chamber.

It can be seen that the pressure differential regularly increases as compressor power is rising. The results of the experiment also reflect a tendency to increase the pressure differential with a decrease in the absolute static air pressure in the pressure chamber.

Table 4 and Fig. 9 represent the readings of the anemometer installed opposite the outlet slit of the acceleration channel at different values of the drive power of the separator compressor and static air pressure inside the pressure chamber.

One can see that there is a clear dependence of the reference anemometer readings on the drive power of the separator compressor. At the same time, the change in static air pressure does not significantly affect the reference anemometer readings, which attests that it is impossible to control the gas velocity according to the reference anemometer readings without correction for static pressure.

It should be noted that the anemometer sensor, calibrated for each value of the static gas pressure, installed and fixed strictly at one point, can be used for on-line gas velocity testing at constant static pressure.

A freeze frame of the tracer particle video filming is shown in Fig. 10. When playing video files at the speed 40 times lower than the speed at which they were shot (24 frames/s versus 960 frames/s), it is possible to visually track the movement of tracer particles. The marks applied to the transparent wall of the acceleration channel allow us to estimate the distance passed by each particle for each separate frame. The length of the strokes that the particles leave when recording at a known shutter speed $(1 / 1000 \mathrm{~s})$ also allows us to estimate rate of movement of the particles.

The linear velocity of the tracer particles is calculated based on the results of the video sequence analysis according to the formula:

$$
u=\sum_{i=1}^{S}\left(L 2_{i}-L 1_{i}\right) \cdot \frac{960}{N},
$$

where $i$ is a number of a particle from a random sample; $S$ is the sample size (varied within 20-30 according to the standard deviation nonexceedance condition for the point values of the calculated velocity equal to $10.0 \%$ rel.); $L 2$ is the final particle coordinate, $\mathrm{m} ; L 1$ is the initial particle coordinate, $\mathrm{m} ; N$ is the number of frames during which the particle passed the distance $L 1-L 2$.

Table 5 and Fig. 11 show the values of the measured velocity of the tracer particles at different values of the drive power of the separator compressor and static air pressure in the main volume of the pressure chamber.

One can recognize that the air velocity increases as compressor power rises, and there is a tendency of the air velocity increase with a decrease in static pressure.

In the author's opinion, a disproportionately strong increase in the measured velocity with an increase in the compressor power to $1.6 \mathrm{~kW}$ is connected with a change 


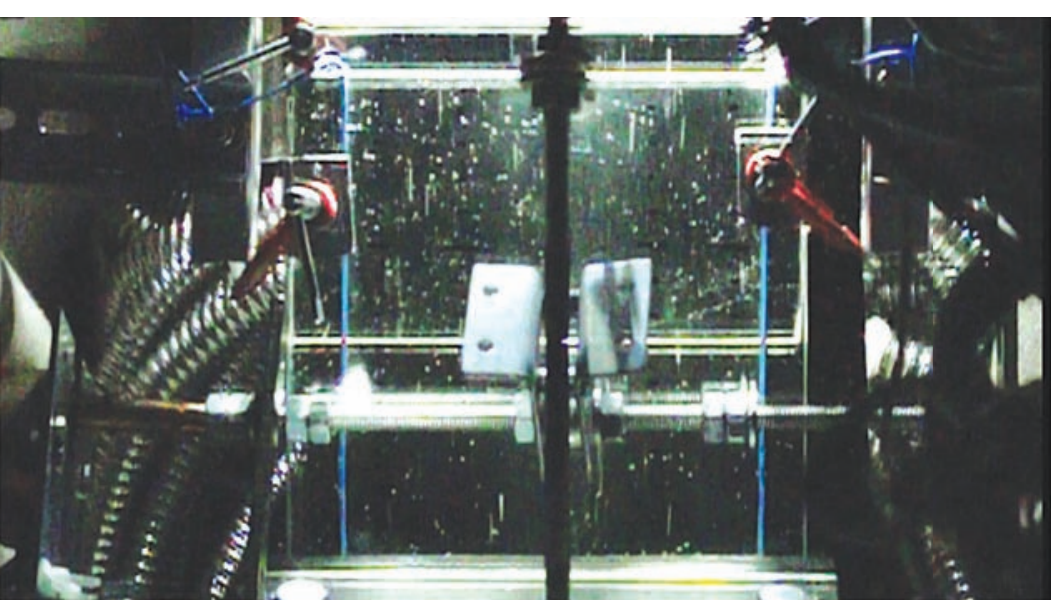

Fig. 10. Typical observed pattern of particle motion in the acceleration channel

Table 5

Measured velocity of the anemometer particles

\begin{tabular}{|c|c|c|c|c|}
\hline $\begin{array}{c}\text { Experiment } \\
\text { number }\end{array}$ & $\begin{array}{c}\text { Absolute pressure in the } \\
\text { pressure chamber, kPa }\end{array}$ & $\begin{array}{c}\text { Compressor } \\
\text { power, kW }\end{array}$ & $\begin{array}{c}\text { Measured } \\
\text { velocity, m/s }\end{array}$ & Re \\
\hline 1 & $20.0 \pm 1.0$ & 0.2 & 9.47 & 351 \\
\hline 2 & $20.0 \pm 1.0$ & 0.4 & 14.39 & 549 \\
\hline 3 & $60.0 \pm 1.0$ & 0.3 & 11.03 & 1254 \\
\hline 4 & $60.0 \pm 1.0$ & 0.7 & 17.45 & 1998 \\
\hline 5 & $60.0 \pm 1.0$ & 1.1 & 22.13 & 2572 \\
\hline 6 & $96.5 \pm 1.0$ & 0.4 & 10.30 & 1893 \\
\hline 7 & $96.5 \pm 1.0$ & 0.9 & 17.29 & 3210 \\
\hline 8 & $96.5 \pm 1.0$ & 1.4 & 41.20 & 7685 \\
\hline
\end{tabular}

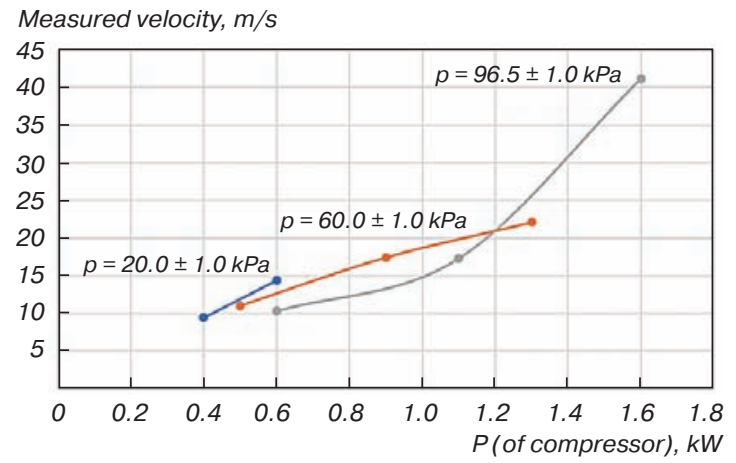

Fig. 11. Dependence of the measured velocity $(\mathrm{m} / \mathrm{s})$ on the drive power of the separator compressor $(\mathrm{kW})$ at different absolute values of static air pressure in the main volume of the pressure chamber in the airflow regime in the acceleration channel, namely, with its transition to a turbulent area. At an air pressure close to atmospheric and a power of 0.9 and $1.4 \mathrm{~kW}$, as can be seen from Table 5, the Reynolds number is, respectively, 3210 and 7685 .

\section{Conclusions}

A comparison of the air velocity measured instrumentally and the velocity calculated by hydrodynamic formulas is given in Table 6.

It can be seen that the ratio of the actually measured air velocity to that calculated by hydrodynamic formulas is in the range $[0.8 ; 1.2]$ at an average rarefaction (absolute pressure is about $60 \mathrm{kPa}$ ), as well as at a pressure close to atmospheric $(96.5 \mathrm{kPa})$, provided that the transition of the airflow regime through the acceleration channel into the turbulent area is prevented.

According to the author's assumption, the mass of tracer particles and their sliding friction against the lower wall of the acceleration channel begins to affect at low absolute air pressure (at the level of $20 \mathrm{kPa}$ ) and, as a consequence, its low density. In this regard, the experimental method significantly, by more than $20 \%$ rel., underestimates the air velocity value.

The overall high convergence of the calculated and experimental methods for measuring air velocity allows us to draw the following conclusions about the procedure of measuring and testing the working gas velocity during gasdynamic separation:

1. For each gas, it is necessary to carry out an actual measurement of linear velocity of its laminar flow at different values of compressor power and absolute pressure.

2. Based on the results of the measurements, it is necessary to compile a table of results and further determine the gas velocity according to this table, depending on the two above-mentioned regimes $(\mathrm{P}, \mathrm{p})$.

3. The correctness of measurements should be checked during the processing of the results with simultaneous

Table 6

Results of calculation and instrumental measurement of air velocity at different static pressure

\begin{tabular}{|c|c|c|c|c|c|c|c|}
\hline $\begin{array}{l}\text { Experiment } \\
\text { number }\end{array}$ & $\begin{array}{l}\text { Absolute pressure in the } \\
\text { pressure chamber, } \mathrm{kPa}\end{array}$ & $\begin{array}{l}\text { Compressor } \\
\text { power, kW }\end{array}$ & $\begin{array}{c}\text { Calculated } \\
\text { air velocity, } \\
\text { m/s }\end{array}$ & $\begin{array}{c}\text { Measured } \\
\text { average } \\
\text { air velocity, } \mathrm{m} / \mathrm{s}\end{array}$ & $\begin{array}{l}\text { The measured } \\
\text { to calculated } \\
\text { velocity ratio }\end{array}$ & Re calc. & Re act. \\
\hline 1 & $20.0 \pm 1.0$ & 0.2 & 21.0 & 9.47 & 0.45 & 778 & 351 \\
\hline 2 & $20.0 \pm 1.0$ & 0.4 & 28.0 & 14.39 & 0.51 & 1070 & 549 \\
\hline 3 & $60.0 \pm 1.0$ & 0.3 & 13.3 & 11.03 & 0.83 & 1508 & 1254 \\
\hline 4 & $60.0 \pm 1.0$ & 0.7 & 18.3 & 17.45 & 0.95 & 2092 & 1998 \\
\hline 6 & $96.5 \pm 1.0$ & 0.4 & 10.5 & 10.30 & 0.98 & 1934 & 1893 \\
\hline 7 & $96.5 \pm 1.0$ & 0.9 & 15.2 & 17.29 & 1.14 & 2817 & 3210 \\
\hline 8 & $96.5 \pm 1.0$ & 1.4 & 18.2 & 41.20 & 2.26 & 3404 & 7685 \\
\hline
\end{tabular}


calculating the speed according to the formulas of hydrodynamics.

4. For on-line testing and detection of emergency conditions (leakages, receiver depressurization, clogging of the upper stage channel with material) it is necessary to carry out monitoring of the air velocity constant value determined by reference anemometer calibrated for each value of static gas pressure.

\section{References}

1. Turning Moon Dust into Oxygen. The European Space Agency, 27 Nov. 2020. URL: https://www.esa.int/Science_Exploration/ Human_and_Robotic_Exploration/Turning_Moon_dust_into_ oxygen (accessed: 15.11.2021).

2. Jones A. China is Working on a Lander for Human Moon Missions. Space News. Aug. 9, 2021. https://spacenews.com/ china-is-working-on-a-lander-for-human-moon-missions/ (accessed: 15.11.2021).

3. Bandyopadhyay S. Lunar Crater Radio Telescope (LCRT) on the Far-Side of the Moon. NASA, Apr. 7, 2020. Avilable at: https://nasa.gov/directorates/spacetech/niac/2020_Phase_I_ Phase_II/lunar_crater_radio_telescope/ (accessed: 15.11.2021).

4. Ignatova A. M., Ignatov M. N. Use of Resources for Regolith Exploration of the Lunar Surface. International Journal of Experimental Education. 2013. No. 11-2. pp. 101-110.
5. Barsukov V. L. Lunar Soil from the Mare Crisium. Moscow : Nauka, 1980. 360 p.

6. Samygin V. D., Filippov L. O., Shekhirev D. V. Ores Beneficiation Basics. Moscow: "Alteks", 2003. 304 p.

7. Tyukin A. P. Developing a Combination Concentration Process for Granular Material Involving Aerodynamic and Shock Separation. Dissertation ... of Candidate of Technical Sciences. Moscow : MISiS, 2013. 151 p.

8. Tyukin A. P., Yushina T. I. Mathematical Modelling of Gas-Dynamic Separation Processes. Tsvetnye Metally. 2020. No 7. pp. 9-17. DOI: 10.17580/tsm.2020.07.01

9. Geyer V. G., Dulin V. S., Zarya A. N. Hydraulics and hydraulic drive. Moscow: Nedra, 1991. 331 p.

10. Porolo L. V. Air-Gas Liquid Lifts (Ergazlifts). Basics of Theory and Calculation Methods. Moscow: "Mashinostroenie", 1969. 160 p.

11. Vilner Ya. M., Kovalev Ya. T., Nekrasov B. B. Reference Manual on Hydraulics, Hydraulic Machines and Hydraulic Drives. Minsk: Vysshaya schola, 1985. 382 p.

12. Idelchik I. E. Handbook of Hydraulic Resistance. Coefficients of Local Resistance and of Friction. National Science Foundation, Washington, D.C. Nov. 1986. 517 p.

13. ISO 12232:2019. Photography - Digital still cameras Determination of exposure index, ISO speed ratings, standard output sensitivity, and recommended exposure index. ICS $>37>$ $>37.040>37.040 .10$. Published Date: Feb. 2019. 21 p. 